\title{
Resveratrol enhances decidualization of human endometrial stromal cells
}

\author{
Ana Cecilia Mestre Citrinovitz, Laila Langer, Thomas Strowitzki and Ariane Germeyer \\ Department of Gynecological Endocrinology and Fertility Disorders, Women's Hospital, Ruprecht-Karls University \\ of Heidelberg, INF 440, Heidelberg, Germany \\ Correspondence should be addressed to A C Mestre Citrinovitz; Email: mestre.ibyme@gmail.com
}

\begin{abstract}
The differentiation of endometrial stromal cells (ESC), named decidualization, is essential to regulate trophoblast invasion and to support pregnancy establishment and progression. Decidualization follows ESC proliferation and it has been described that cell cycle arrest contributes to a proper decidualization. Interestingly, resveratrol, a natural compound derived from grapes with antioxidant properties, has been widely studied in relation to endometrial health. However, little is known about the effect of resveratrol supplementation during decidualization. Therefore, in this study we evaluate the effect of resveratrol supplementation during decidualization. We used primary and immortalized human ESC and we decidualized them in vitro with a decidualization cocktail containing medroxyprogesterone acetate, estradiol and 8-Bromo-cyclic AMP. Pre-decidualized cells were further treated with the decidualization cocktail supplemented with resveratrol. Our results show that resveratrol supplementation increased, in a dosedependent manner, the expression levels of prolactin and IGFBP1 (RT-PCR and ELISA), indicating an enhanced in vitro decidualization of human ESC. This enhanced decidualization was accompanied by a decrease in cell proliferation (crystal violet and CellTiter proliferation assay) and by changes in the mRNA levels of key cell cycle regulators (RT-PCR). Furthermore, resveratrol supplementation seemed to enhance decidualization by reinforcing the effect of the decidualization cocktail. We believe that resveratrol could to be an effective supplementation to reinforce hormone action during human ESC decidualization and that further insights into resveratrol action and its interaction with estradiol and progesterone signaling pathways could facilitate the identification of new therapeutic strategies for the improvement of women's health.

Reproduction (2020) 159 453-463
\end{abstract}

\section{Introduction}

During the menstrual cycle, the endometrium undergoes cyclic rounds of cell proliferation, differentiation and death. After ovulation, during the secretory phase of the menstrual cycle, the differentiation of human ESC takes place. This differentiation process is known as decidualization and is a tightly regulated process. Decidualization compromises of a profound change in cell morphology and function that is accompanied by a complete reprogramming of gene and protein expression (Ramathal et al. 2010). This change in cell identity is critical to ensure proper feto-maternal interactions and to guide placentation, ensuring a healthy pregnancy (Strowitzki et al. 2006, Gellersen \& Brosens 2014).

It has been widely described that cell cycle arrest is an important event related to cell differentiation (Reichert \& Eick 1999, Myster \& Duronio 2000, Kwon et al. 2016, Ruijtenberg \& van den Heuvel 2016, Soufi \& Dalton 2016). Cell cycle progression is tightly regulated by the coordinated action of cyclin-dependent kinases (CDK). CDK activity is regulated by cyclins and CDK inhibitors (CDKI) that are expressed in a timely manner during the cell cycle. In relation to endometrial function, it has been demonstrated that the expression of several cell cycle regulators is modified during decidualization and that ESC exit the cell cycle before differentiating (Takano et al. 2007, Logan et al. 2012). In particular, previous findings have determined that ESC are arrested at the G0/G1 checkpoint during decidualization (Wang et al. 2018). The activation and crosstalk of progesterone (P) and cyclic AMP (cAMP) signaling pathways regulate the changes in cell cycle progression (Gellersen \& Brosens 2003). Furthermore, $P$ and cAMP are key to drive the whole protein and gene expression reprogramming of ESC during decidualization (Gellersen \& Brosens 2014).

Defective decidualization has been associated with impaired pregnancy establishment and progression (Strowitzki et al. 2006). For example, it has been studied that impaired decidualization could have an important role during the onset of preeclampsia, a placenta-related pathology (Garrido-Gomez et al. 2017). Moreover, it has been demonstrated that ESC derived from women with endometriosis, a benign disorder defined by the presence of endometrial tissue outside the uterine cavity, have reduced expression of decidualization 
markers compared to ESC derived from women without endometriosis (Klemmt et al. 2006, Aghajanova et al. 2009).

Interestingly, resveratrol (R) (3,4',5-trihydroxy-transstilbene), a natural polyphenolic compound derived from some fruits and vegetables, has been widely studied in relation to reproductive health. Several authors have described the beneficial properties of $\mathrm{R}$ in relation to the inhibition of cell proliferation and migration and the reduction of oxidative stress and pointed to $R$ as a possible drug for the treatment of endometriosis and preeclampsia (Ricci et al. 2013, Zou et al. 2014, Hannan et al. 2017, Kolahdouz Mohammadi \& Arablou 2017). Furthermore, it has been shown that $\mathrm{R}$ exerts a positive effect over fertility in relation to ovarian aging in mice, as the long-term oral administration of $\mathrm{R}$ improved the number and quality of oocytes, allowing old, female mice to retain more capacity to reproduce (Liu et al. 2013, Ortega \& Duleba 2015).

Due to the described beneficial effects of $R$ in women's reproductive health, we were interested in evaluating the effect of $\mathrm{R}$ supplementation during decidualization. Therefore, the aim of this study was to evaluate the effect of resveratrol supplementation during in vitro decidualization of human ESC. Herein, we describe that resveratrol supplementation together with medroxyprogesterone acetate (MPA), estradiol and 8-Bromo-cAMP (8-Br-cAMP) enhances the decidualizing effect of these drugs leading to a higher expression of prolactin (PRL) and Insulin-like growth factor binding protein 1 (IGFBP1). In addition, we describe that $R$ supplementation enhances the inhibition in cell proliferation that the decidualization program requires, probably contributing additionally, this way, to ESC differentiation.

\section{Materials and methods \\ Ethical approval}

This study protocol was approved by the ethical committee (S-239/2005) of the Ruprecht-Karls University of Heidelberg. Biopsies were taken after written informed consent was obtained from all participants.

\section{Cell isolation and culture}

Primary cells

Endometrial biopsies from mid-late proliferative phase were obtained from healthy regularly-cycling women $(34.4 \pm 1.1$ years old) without hormonal therapies, no endometrial abnormalities and no endometriosis at diagnostic laparoscopy. Endometrial biopsies were cut into small pieces and digested with collagenase (Gibco) and hyaluronidase (Sigma-Aldrich) at $37^{\circ} \mathrm{C}$ for 60 to $90 \mathrm{~min}$. To remove epithelial cells, cell suspensions were filtered through a $40 \mu \mathrm{m}$ mesh. Isolated primary human ESC (HESC) were cultured in a 3:1 mixture of DMEM (Cat Number 21063, Gibco) and MCDB-105 (Cat
Number M6395, Sigma-Aldrich) without phenol red and supplemented with $10 \% \mathrm{v} / \mathrm{v}$ charcoal/dextran treated fetal bovine serum (FBS) (HyClone, GE Healthcare Europe). Cell passages number two to four were used for the experiments described subsequently.

\section{Cell line}

Immortalized human ESC (t-HESC) (Krikun et al. 2004) were purchased from LGC (ATCC CRL-4003, LGC Standards GmbH, Wesel, Germany) and cultivated in DMEM-F12 (Cat Number D2906, Sigma-Aldrich) without phenol red according to ATCC recommended instructions.

\section{In vitro decidualization and resveratrol supplementation}

Cells were trypsinized, counted and plated in 6-well plates $(150,000$ cells/well for HESC and 100,000 cells/well for t-HESC). After 24-48 h, when cells had reached $80-90 \%$ confluency, medium was removed and cells were rinsed with PBS. Then, $2 \mathrm{~mL}$ of fresh $2 \% \mathrm{v} / \mathrm{v}$ FBS medium supplemented with a decidualization cocktail (DC) containing $1 \mu \mathrm{M}$ medroxyprogesterone acetate (MPA), $10 \mathrm{nM}$ estradiol (E) and $0.375 \mathrm{mM}$ 8-Br-cAMP ( $\mathrm{D}$, decidualized) or vehicle solutions (ND, non-decidualized) were added to each well. At days 3 and 5 of the decidualization treatment, medium was changed and different doses of resveratrol (0 vehicle treated; 6.25; $12.5 ; 25 ; 50 \mu \mathrm{M})$ were added to each well. Only cells treated with the DC were supplemented with resveratrol. After 2 and 4 days of resveratrol supplementation (days 5 and 7 of the decidualization protocol), cell supernatants (SN) were collected for the quantification of prolactin levels and cell samples were collected in TRIzol reagent (Invitrogen, Life Technologies $\mathrm{GmbH}$ ) for total RNA extraction or in RIPA buffer for protein detection by Western blot. For microtubuleassociated protein light chain 3 B (LC3B) detection by Western blot, chloroquine $(25 \mu \mathrm{M})$ or vehicle solution were added to the culture medium $8 \mathrm{~h}$ before harvesting the cells. Drugs used are listed in Supplementary Table 1 (see section on supplementary materials given at the end of this article).

\section{Prolactin quantification}

Prolactin from cell culture supernatant was quantified at the central laboratory of Heidelberg University Clinic using a double sandwich ELISA assay (Siemens Diagnostics) according to the manufacturer's instruction.

\section{Gene expression assays}

Gene expression assays were performed exactly as previously described (Mestre Citrinovitz et al. 2019). Primers used are listed in Supplementary Table 1. RT-PCR reactions were run in the Fast Forward 7500 real-time PCR-system (Applied Biosystems, Life Technologies $\mathrm{GmbH}$ ). RT-PCR results were analyzed according to the $\Delta \Delta$ Ct method (Livak \& Schmittgen 2001), and RPLPO was used as internal control for normalization of Ct values. Normalized $\mathrm{Ct}$ values $(\Delta \mathrm{Ct})$ were used for statistical analysis. 


\section{Proliferation assays}

t-HESC and HESC were trypsinized, counted and plated in 96-well plates at a density of 1000 cells/well. After 24-48 h, medium was removed and $100 \mu \mathrm{L}$ of fresh $2 \% \mathrm{v} / \mathrm{v}$ FBS medium containing the DC were added to each well. At days 3 and 5 of the decidualization treatment, medium was changed and cell cultures were supplemented with or without $25 \mu \mathrm{M}$ of resveratrol. After 4 days of resveratrol supplementation (day 7 of the decidualization protocol), cell proliferation was evaluated by crystal violet (CV) staining and by CellTiter 96 Aqueous One Solution Cell Proliferation assay (Promega Corporation). Proliferation assays were performed according to manufacturer's instructions and exactly as previously described (Feoktistova et al. 2016, Mestre Citrinovitz et al. 2019).

\section{Western blot analysis for protein detection}

LC3B and p62 detection by Western blot was performed exactly as previously described (Mestre Citrinovitz et al. 2019). Antibodies used and working dilutions are listed in Supplementary Table 1.

\section{Statistical analysis}

Paired Student's $t$-test and one-way ANOVA followed by Dunnett's Multiple Comparison Test were used for statistical analysis. Differences between treatments were considered significant when $P<0.05$. Statistical analysis was carried out using GraphPad Prim 4.0 (GraphPad Software Inc.).

\section{Results}

\section{Resveratrol supplementation enhances HESC decidualization}

As a first approach to evaluate the effect of $R$ supplementation during the decidualization of HESC, we measured the expression level of PRL and IGFBP1, two well-known decidualization markers (Brosens et al. 1996), in HESC treated with the DC supplemented with different doses of R. As expected, after 5 and 7 days of DC treatment, PRL protein levels and PRL and ICFBP1 mRNA levels were increased in decidualized (D) cells compared to non-decidualized (ND) cells (Supplementary Fig. 1). R supplementation further increased PRL protein levels and PRL and IGFBP1 mRNA levels in a dose-dependent manner compared to $0 \mu \mathrm{M}$ of $R$ (Fig. $1 \mathrm{~A}$ and $\mathrm{B}$ ).

Then, we decided to evaluate the changes in mRNA expression of other secreted factors known to be regulated during decidualization: IL-8, a cytokine related to trophoblast invasion, and VEGFA, an important proangiogenic factor. The mRNA levels of both genes were increased during decidualization (Supplementary Fig. 1). However, in response to $R$ supplementation IL$8 \mathrm{mRNA}$ levels were decreased in a dose-dependent manner, while VEGFA mRNA levels were slightly diminished only by $50 \mu \mathrm{M}$ of $\mathrm{R}$ (Fig. 2A).
To move forward in the description of the effect of $\mathrm{R}$ supplementation during the decidualization, we decided to evaluate the mRNA levels of HOXA10 (Qian et al. 2005, Godbole et al. 2017), FOXO1 (Kajihara et al. 2013) and NOTCH1 (Afshar et al. 2012), three genes tightly related to gene expression regulation
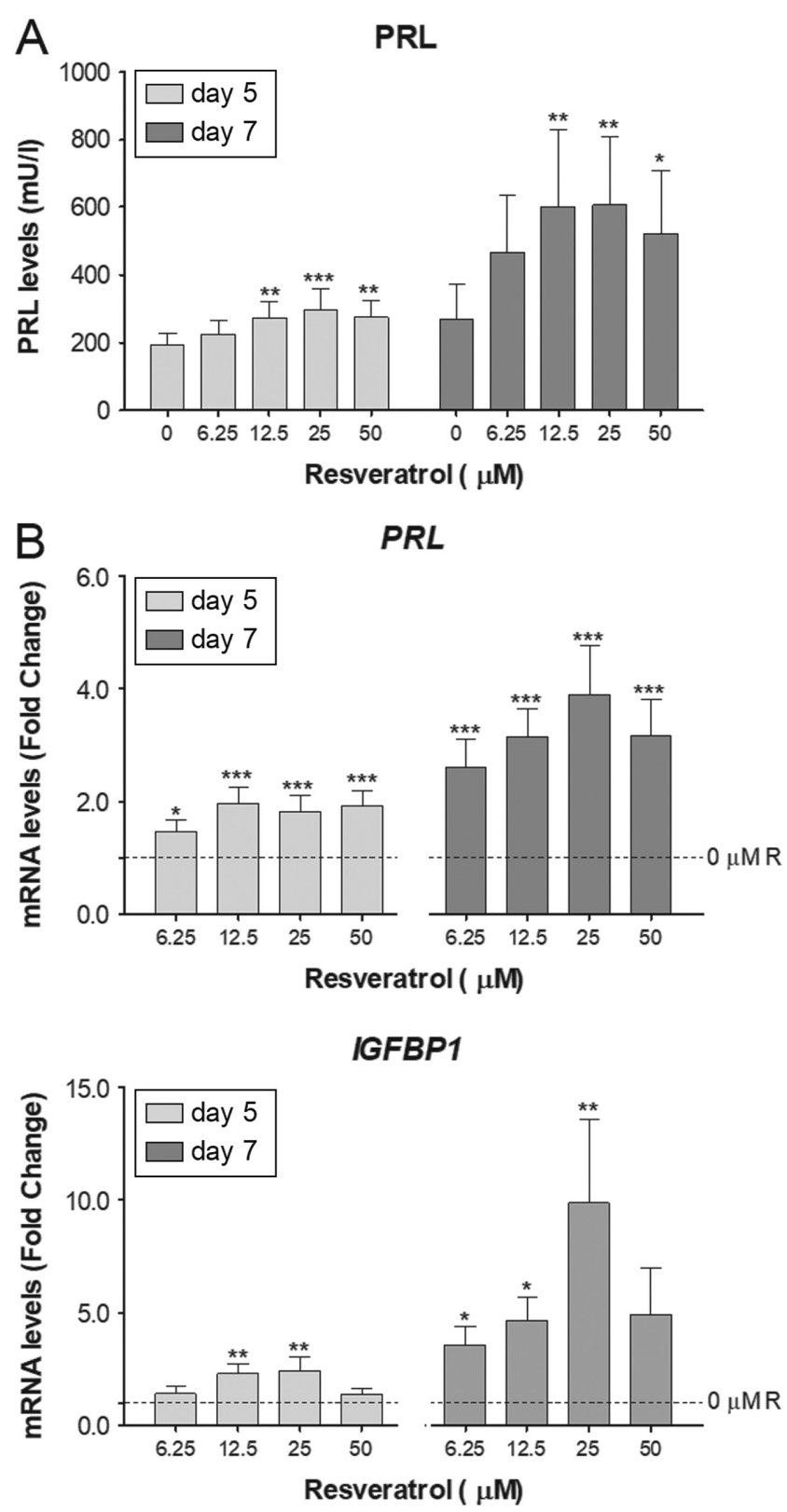

Figure 1 Resveratrol supplementation increases PRL and IGFBP1 expression during in vitro decidualization of HESC. HESC were treated with DC for 3 days and then treated with DC plus different concentrations of $\mathrm{R}$ for 2 or 4 more days. (A) PRL levels in cell culture supernatant $(n=5)$. Data represent mean $\mathrm{mU} / \mathrm{I} \pm$ S.E.M. (B) $P R L$ and IGFBP1 mRNA expression levels $(n=5)$. Data represent mean fold change compared to $0 \mu \mathrm{M} \mathrm{R}$ (dotted line, fold change $=1.0) \pm$ S.E.M. ${ }^{*} P<0.05 ;{ }^{* *} P<0.01 ; * * * P<0.001$ (compared to $0 \mu \mathrm{M}$ of R). DC: decidualization cocktail; R: resveratrol; light grey: day 5; dark grey: day 7 . 

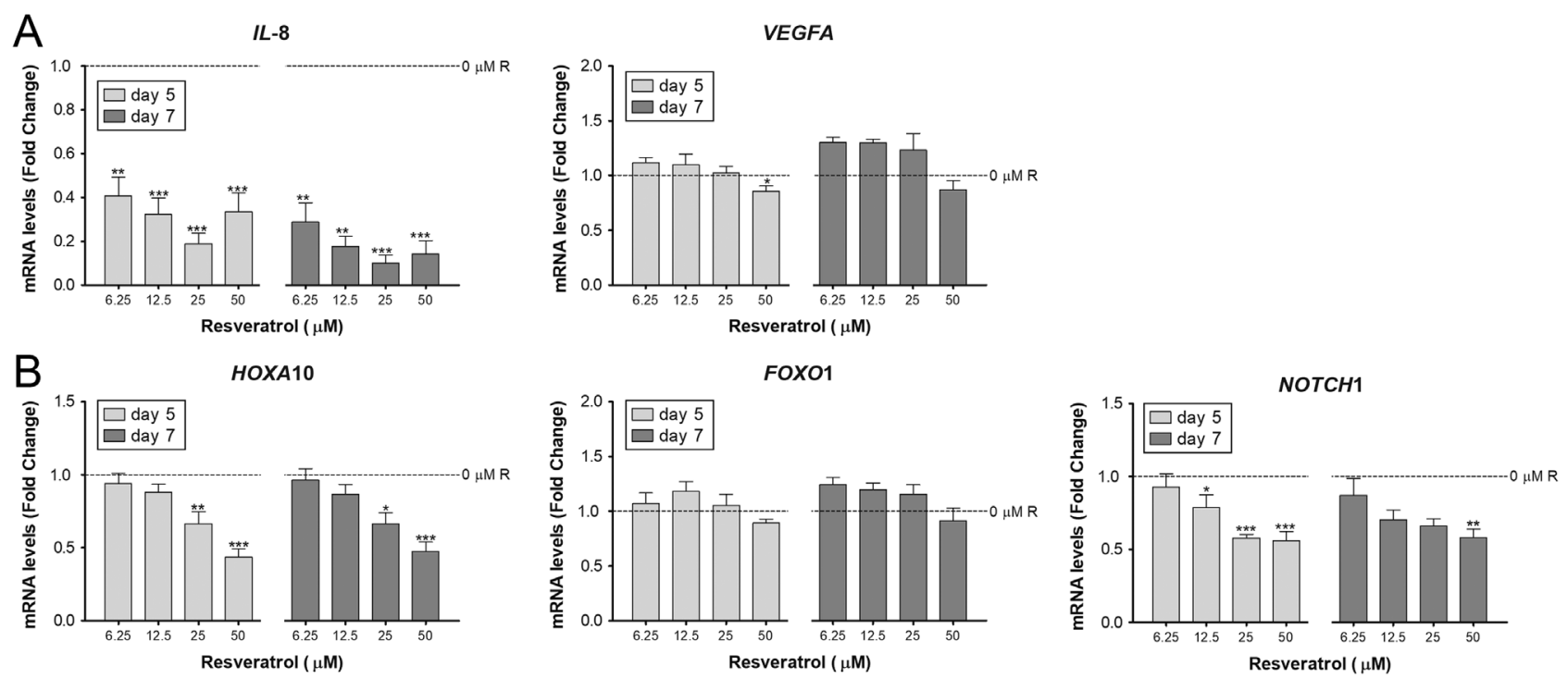

Figure 2 Resveratrol supplementation during in vitro decidualization of HESC regulates the mRNA expression of several genes associated with decidualization. HESC were treated with DC for 3 days and then treated with DC plus different concentrations of R for 2 or 4 more days. (A) Secreted factors: IL-8 and VEGFA mRNA expression levels $(n=5)$. (B) Genes associated to gene expression regulation and cell signaling: HOXA10, FOXO1 and NOTCH1 mRNA expression levels $(n=5)$. Data represent mean fold change compared to $0 \mu \mathrm{M} R \pm$ S.E.M. ${ }^{*} P<0.05$; ${ }^{* *} P<0.01$; ${ }^{* * *} P<0.001$ (compared to $0 \mu \mathrm{M}$ of $\mathrm{R}$, dotted line, fold change=1.0). DC: decidualization cocktail; R: resveratrol; light grey: day 5 ; dark grey: day 7.

and to cell signaling during decidualization (Fig. 2B and Supplementary Fig. 1). HOXA10 and NOTCH1 mRNA expression was decreased in response to the DC. In addition, HOXA10 and NOTCH1 mRNA levels were further decreased by $\mathrm{R}$ supplementation in a dose-dependent manner. In contrast, FOXO1 mRNA levels were increased during HESC decidualization (Supplementary Fig. 1), and R supplementation had no additional effect (Fig. 2B).

These results indicatethat $\mathrm{R}$ supplementation reinforces the effect of DC over HESC during decidualization. This increased decidualization was confirmed by the further increased expression of PRL and IGFBP1 upon $\mathrm{R}$ supplementation. In addition, HOXA10 and NOTCH1 mRNA levels, decreased in response to the DC, were further decreased by R supplementation.

\section{t-HESC respond similar to HESC when supplemented with resveratrol during decidualization}

Primary cells isolated from patients' biopsies are an excellent and widely used experimental model to study cellular response to different drugs and to uncover the signaling pathways involved in that response. However, primary cells are a limited source and the use of stablished cell lines helps to overcome this issue. Therefore, we decided to evaluate if t-HESC, a cell line that has a normal respond to decidualization stimulation (Krikun et al. 2004), can be used as an experimental model for the study of the effect of $\mathrm{R}$ supplementation during decidualization.
To compare both cell types in relation to their response to $\mathrm{R}$ supplementation, we treated $\mathrm{t}-\mathrm{HESC}$ as described for HESC and we measured the changes in PRL and IGFBP1 expression levels. We decided to measure only at day 5 of the decidualization treatment, as the effect of $R$ was already evident by that time point in HESC (Fig. 1). After 5 days of decidualization treatment, PRL protein as well as PRL and IGFBP1 mRNA levels were increased in D cells compared to ND cells (Supplementary Fig. 2). As seen for HESC, R supplementation in t-HESC increased the expression levels of PRL protein and PRL and IGFBP1 mRNA in a dose-dependent manner compared to $0 \mu \mathrm{M}$ of R (Fig. 3A and B).

To further compare the effect of $R$ supplementation over t-HESC and HESC decidualization, we evaluated the expression levels of HOXA10, FOXO1 and NOTCH1 in t-HESC (Fig. 3C and Supplementary Fig. 2). As seen for HESC, the expression levels of HOXA1O and NOTCH1 in $\mathrm{t}$-HESC were decreased in response to the DC. However, HOXA10 and NOTCH1 mRNA levels were not further modified by R supplementation. Also, as seen for HESC, mRNA levels of FOXO1 were increased by the DC in t-HESC and were not further modified by $\mathrm{R}$ supplementation.

These results indicate that $\mathrm{R}$ supplementation reinforces the effect of DC over t-HESC during decidualization, confirmed by the increased expression of PRL and IGFBP 1 upon $\mathrm{R}$ supplementation. These results additionally indicate that t-HESC are a useful experimental model to evaluate the effect of $R$ supplementation during human endometrial stromal cells differentiation; however, 

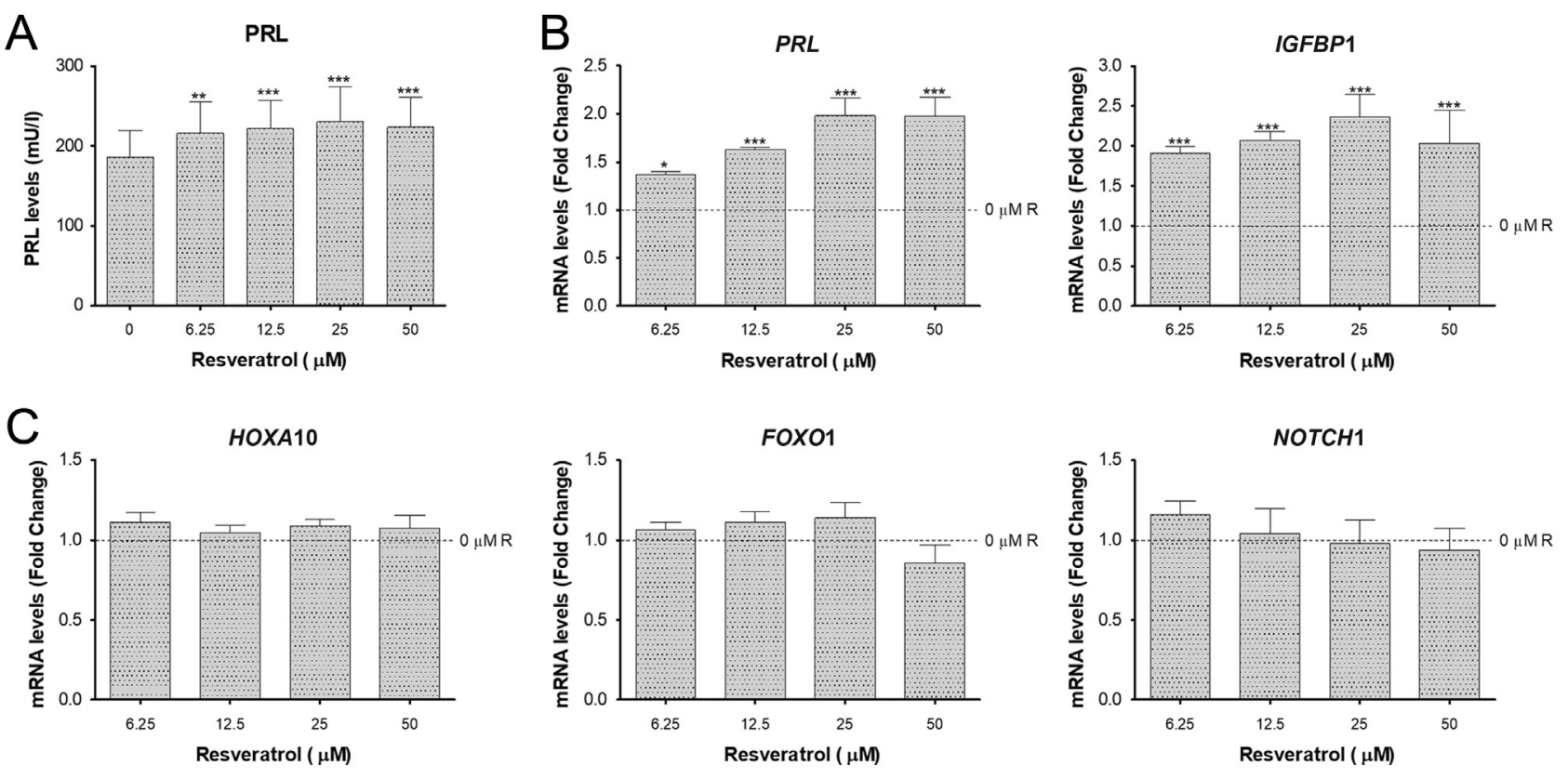

Figure 3 Resveratrol supplementation increases PRL and IGFBP1 expression during in vitro decidualization of t-HESC. t-HESC were treated with DC for 3 days and then treated with DC plus different concentrations of R for 2 more days. (A) PRL levels in cell culture supernatant $(n=4)$.

Data represent mean $\mathrm{mU} / \mathrm{I} \pm$ S.E.M. (B) $P R L$ and $/ G F B P 1$ mRNA expression levels $(n=4)$. (C) Genes associated to gene expression regulation and cell signaling: HOXA10, FOXO1 and NOTCH1 mRNA expression levels $(n=4)$. For PCR (B and C), data represent mean fold change compared to $0 \mu \mathrm{M}$ $\mathrm{R}$ (dotted line, fold change $=1.0) \pm$ S.E.M. ${ }^{*} P<0.05 ; * P<0.01$; ${ }^{* * *} P<0.001$ (compared to $0 \mu \mathrm{M}$ of R). DC: decidualization cocktail; R: resveratrol.

cautioun should be taken when evaluating the signaling pathways involved in this decidualization reinforcement.

\section{Resveratrol supplementation reduces cell proliferation during t-HESC and HESC decidualization}

As cell differentiation is tightly related to cell proliferation and because $\mathrm{R}$ has been described to inhibit cell proliferation in a wide variety of cell types (Cucciolla et al. 2007, Delmas et al. 2011, Ko et al. 2017, Kolahdouz Mohammadi \& Arablou 2017), we decided to evaluate if the enhancing effect of $R$ supplementation over t-HESC decidualization could be related to changes in the proliferation capacity of human ESC. Therefore, we evaluated the mRNA levels of KI67, a well-known proliferation marker. The mRNA levels of KI67 were decreased during t-HESC decidualization, and KI67 mRNA expression was further decreased, in a dose-dependent manner, by R supplementation (Fig. 4A and Supplementary Fig. 3).

To quantify the reduction in t-HESC proliferation, we performed the CV staining and CellTiter proliferation assays. For these assays we tested the $25 \mu \mathrm{M}$ dose of $\mathrm{R}$, as it was the lowest dose to achieve a significant reduction of KI67 expression (Fig. 4A) and, also, seemed to be the most effective dose to achieve a positive effect during decidualization. Both proliferation assays showed that the $25 \mu \mathrm{M}$ dose of $\mathrm{R}$ was effective to reduce t-HESC proliferation by 14 and 15\%, respectively (Fig. 4C and D).
Additionally, we tested if $25 \mu \mathrm{M}$ of $\mathrm{R}$ affected the proliferation of HESC during decidualization. We found that KI67 mRNA levels were decreased during HESC decidualization; however, KI67 mRNA expression was not significantly decreased by $\mathrm{R}$ supplementation (Fig. 4B and Supplementary Fig. 3). Moreover, we performed for HESC the CV staining and CellTiter proliferation assays. Both proliferation assays showed that $25 \mu \mathrm{M}$ of $\mathrm{R}$ reduced HESC proliferation by $5 \%$ compared to $0 \mu \mathrm{M} \mathrm{R}$; however, this reduction was only significant for the CV proliferation assay (Fig. 4E and F).

These results indicate that the increased decidualization resulting from $\mathrm{R}$ supplementation is accompanied by a decreased cell proliferation, suggesting a possible functional connection between proliferation, decidualization and the effect of $R$ supplementation.

\section{Resveratrol supplementation during t-HESC and HESC decidualization regulates the $m R N A$ expression of several genes associated with cell cycle progression}

To understand better how cell proliferation was affected by $\mathrm{R}$ supplementation during t-HESC and HESC decidualization, we evaluated the changes in mRNA expression of key genes involved in cell cycle regulation. In particular, we evaluated the expression of CDC2 (CDK1), CDK2 and CDK4, cyclin A1 (CCNA1), B1 (CCNB1) and D1 (CCND1) and p21 and p53 (Figs 5, 6 and Supplementary Fig. 3). 

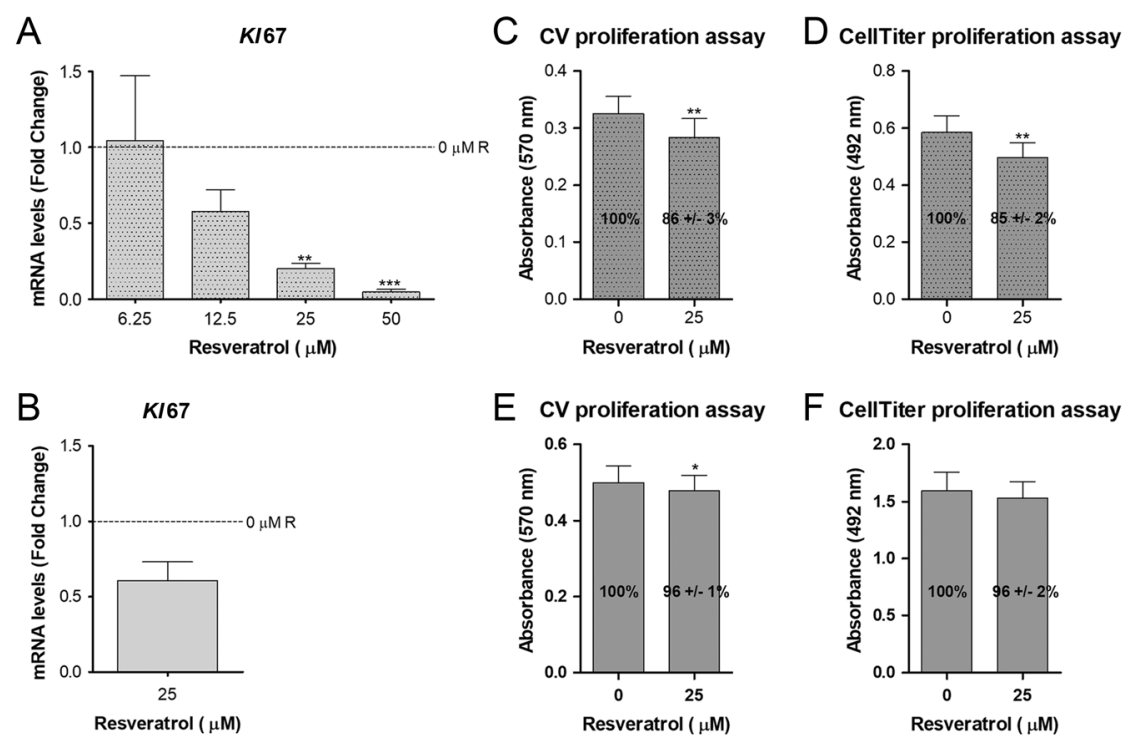

Figure 4 Resveratrol supplementation during in vitro decidualization of t-HESC and HESC decreases cell proliferation. (A and B) t-HESC and HESC were treated with DC for 3 days and then treated with DC plus different concentrations of R for 2 more days. Graph shows KI67 mRNA expression levels (t-HESC: A; $n=4$; HESC: B $n=5$ ). Data represent mean fold change compared to $0 \mu \mathrm{M}$ R \pm S.E.M. ${ }^{* *} P<0.01$; ${ }^{* * *} P<0.001$ (compared to $0 \mu \mathrm{M}$ of $\mathrm{R}$, dotted line, fold change $=1.0$ ). For the proliferation assays, t-HESC and HESC were treated with DC for 3 days and then treated with DC plus $25 \mu \mathrm{M}$ of R or vehicle for 4 more days. (C) t-HESC, Crystal Violet proliferation assay $(n=5)$. (D) t-HESC, CellTiter 96 Aqueous One Solution Cell Proliferation assay $(n=5)$. (E) HESC, Crystal Violet proliferation assay $(n=4)$. (F) HESC, CellTiter 96 Aqueous One Solution Cell Proliferation assay $(n=4)$. Values inside the bar graph represent the percentage of proliferation. Data represent mean absorbance \pm S.E.M. ${ }^{*} P<0.05$; ${ }^{* *} P<0.01$. DC: decidualization cocktail; R: resveratrol; light grey: day 5; dark grey: day 7; dotted bars: t-HESC; empty bars: HESC.

In contrast to what we observed for KI67, the mRNA levels of CCNA1 were increased during t-HESC decidualization and CCNA1 expression showed a tendency to be slightly further increased by $R$ supplementation $(50 \mu \mathrm{M})$. The mRNA levels of CCNB1 and CCND1 and of CDC2,CDK2 and CDK4 were decreased during t-HESC decidualization, and their expression was further decreased by $\mathrm{R}$ supplementation in a dose-dependent manner (Fig. 5 and Supplementary Fig. 3), as seen for KI67. In relation to cell cycle inhibitors, mRNA levels of p53 did not change during t-HESC decidualization; however, $R$ supplementation decreased p53 mRNA levels. The mRNA levels of p21, a p53-dependent gene, were slightly decreased during t-HESC decidualization and had no further changes with R supplementation (Fig. 5 and Supplementary Fig. 3).

During HESC decidualization (Fig. 6 and Supplementary Fig. 3), the mRNA levels of CCNA1 were decreased and R supplementation had not furthered effect. The mRNA levels of CCNB1, CCND1,CDC2 and CDK2 were decreased during HESC decidualization, and their expression was further decreased by $\mathrm{R}$ supplementation. The expression of CDK4 was not modified during HESC decidualization; however, it was decreased by $\mathrm{R}$ supplementation (Fig. 6 and Supplementary Fig. 3). Finally, the mRNA levels of p53 were not changed during HESC decidualization, while R supplementation decreased p53 mRNA levels. The mRNA levels of $p 21$ were highly increased during t-HESC decidualization and were decreased upon $\mathrm{R}$ supplementation.
These results suggest that $R$ supplementation inhibits cell proliferation, mainly by decreasing the expression of positive regulators of cell cycle progression during $\mathrm{t}-\mathrm{HESC}$ and HESC decidualization.

\section{Resveratrol supplementation during t-HESC decidualization does not affect autophagic flux}

Finally, we have recently shown that macroautophagy (herein referred to as autophagy), a highly conserved degradation pathway, is increased during t-HESC decidualization (Mestre Citrinovitz et al. 2019). Furthermore, the transfection of small interference RNA (siRNA) against autophagy related (ATG) proteins 7 and 5 , key proteins involved in the autophagy pathway, led to the impairment of decidualization, highlighting the importance of these proteins and of the autophagy pathway for the proper decidualization of human ESC.

Interestingly, it has been described that $\mathrm{R}$ is capable of activating autophagic flux (Hasima \& Ozpolat 2014). Therefore, we evaluated whether $\mathrm{R}$ supplementation was able to increase autophagic flux during t-HESC decidualization. We measured the protein levels of LC3BII, the lipidated and autophagosome-associated form of $\mathrm{LC} 3 \mathrm{~B}$, in a system were autophagic flux was blocked with chloroquine (CQ) and of p62 in a CQ-free system. Both proteins are degraded when autophagy is active and they are commonly used to evaluate changes in the autophagic flux (Klionsky et al. 2016). In this study, we found no differences in the protein levels of 
A

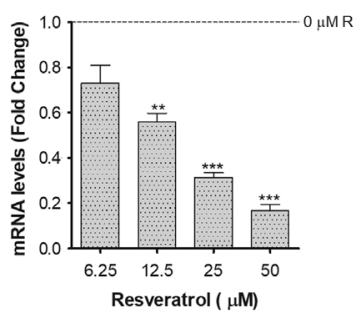

B
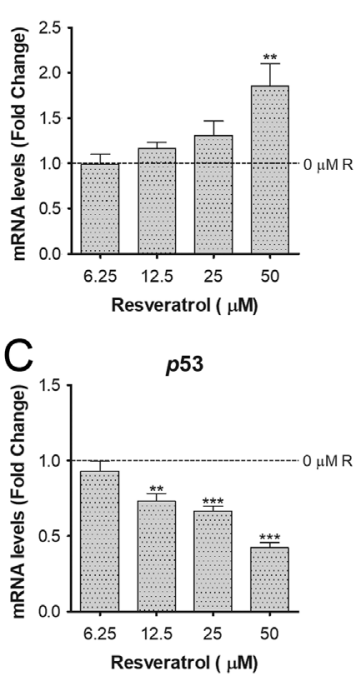

CDK2

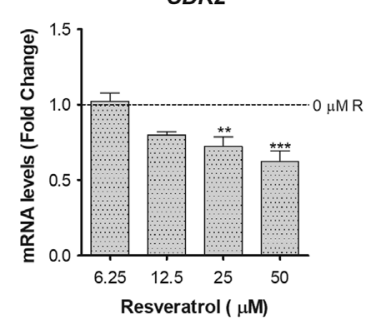

CCNB1

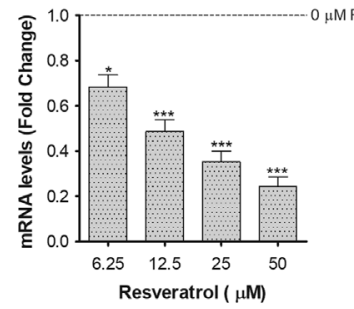

p21

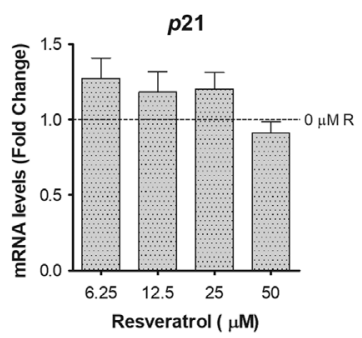

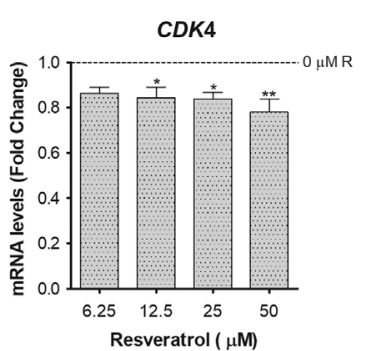

CCND1

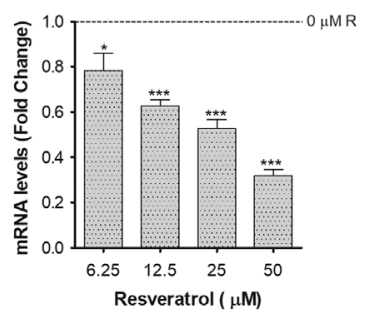

Figure 5 Resveratrol supplementation during in vitro decidualization of t-HESC regulates mRNA expression of several genes associated with cell cycle progression. t-HESC were treated with DC for 3 days and then treated with DC plus different concentrations of $\mathrm{R}$ for 2 more days. Graphs show (A) $C D C 2$, CDK2, CDK4; (B) CCNA1, CCNB1, CCND1 and (C) p53 and p21 mRNA expression levels $(n=4)$. Data represent mean fold change compared to $0 \mu \mathrm{M} \mathrm{R} \pm$ S.E.M. $* P<0.05 ;{ }^{* *} P<0.01$;

${ }^{* * *} P<0.001$ (compared to $0 \mu \mathrm{M}$ of $\mathrm{R}$, dotted line, fold change $=1.0$ ). DC: decidualization cocktail; R: resveratrol.

LCB3II and p62 between $25 \mu \mathrm{M}$ of $\mathrm{R}$ supplementation compared to $0 \mu \mathrm{M}$ (Fig. 7).

These results indicate that the enhanced decidualization resulted from $\mathrm{R}$ supplementation is not accompanied by changes in the autophagic flux.

\section{Discussion}

Our present data demonstrate that $\mathrm{R}$ supplementation during in vitro decidualization of human ESC modifies - in a dose-dependent manner - the expression of several genes related to decidualization. In particular, we observed an increased expression of $P R L$ and IGFBP1, indicating that $\mathrm{R}$ supplementation reinforces decidualization. Furthermore, we detected a decreased expression of key cell cycle regulators and a concomitant reinforcement of the inhibition of cell proliferation stimulated by the DC. In general, we found that $\mathrm{R}$ supplementation boosted the changes in gene expression stimulated in response to the DC, suggesting that $\mathrm{R}$ could be enhancing decidualization by reinforcing DC action in ESC.

\section{Resveratrol and the expression of decidualization- related genes}

Regarding the expression of secreted factors, R supplementation led to a dose-dependent increased expression of PRL and IGFBP1. It has been previously described that the combined addition of PRL and IGFBP1 increases cytotrophoblast invasion (GarridoGomez et al. 2017), indicating that the increased expression of these two secreted factors by decidual cells could contribute on its own to an improved embryo implantation. On the other hand, we found that $\mathrm{R}$ supplementation led to a decreased IL-8 expression counter-acting the increased expression stimulated by the DC. Reduced expression of IL-8 in different cell populations within the decidua has been associated with sporadic miscarriage in relation to normal cases (Pitman et al. 2013). It is important to notice that CD56+ uterine Natural Killer cells, an important endometrial source of growth factors and cytokines, macrophages and CD8+ T lymphocytes are also important decidual sources of IL-8 (De Oliveira et al. 2010, Pitman et al. 2013). Therefore, the decreased expression of IL-8 in ESC generated by $\mathrm{R}$ supplementation does not necessarily mean that trophoblast invasion will be impaired in vivo. Moreover, it has been described that a transient increase of IL-8 expression during early decidualization could explain why more decidualized cells - upon supplementation with $\mathrm{R}$ - express less IL-8 than less decidualized cells supplemented with vehicle (Brighton et al. 2017). Finally, the proangiogenic factor VEGFA was not modified by $R$ supplementation, suggesting that $\mathrm{R}$ may not affect decidual vascularization. 

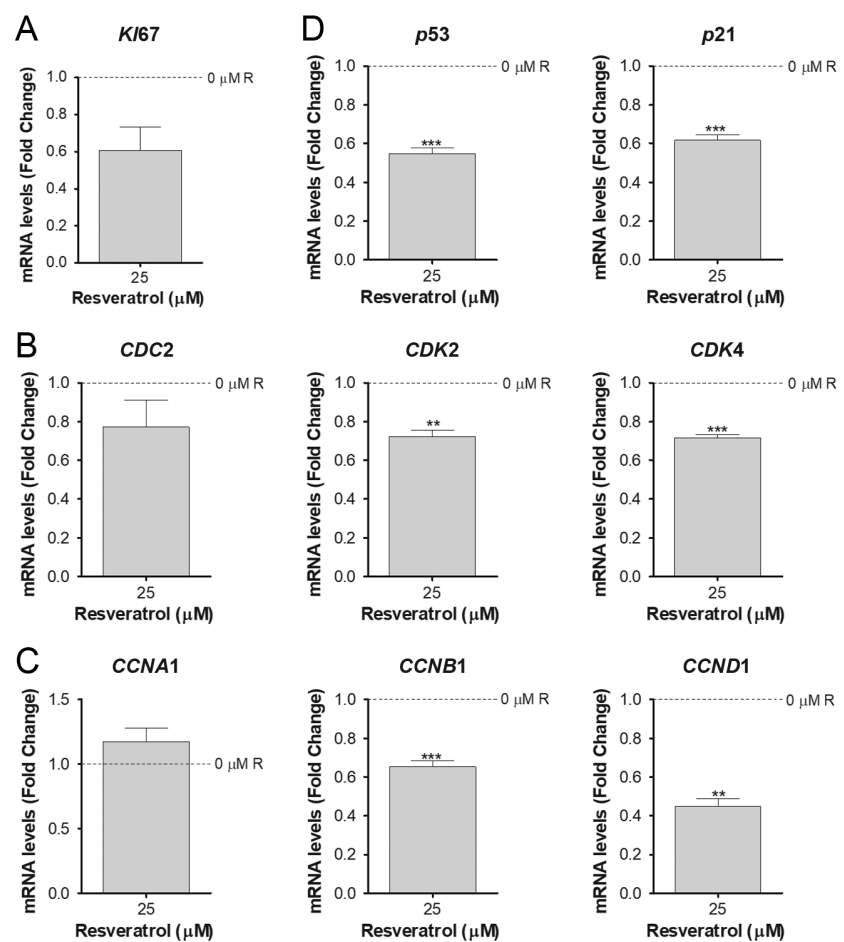

Figure 6 Resveratrol supplementation during in vitro decidualization of HESC regulates mRNA expression of several genes associated with cell cycle progression. HESC were treated with DC for 3 days and then treated with DC plus different concentrations of $\mathrm{R}$ for 2 more days. Graphs show (A) KI67; (B) CDC2,CDK2, CDK4; (C) CCNA1, CCNB1, CCND1 and (D) p53 and p21 mRNA expression levels $(n=5)$. Data represent mean fold change compared to $0 \mu \mathrm{M} \mathrm{R} \pm$ S.E.M. ${ }^{* * P}<0.01 ;{ }^{* * *} P<0.001$ (compared to $0 \mu \mathrm{M}$ of $\mathrm{R}$, dotted line, fold change $=1.0$ ). DC: decidualization cocktail; R: resveratrol.

Regarding the expression of genes involved in cell signaling and gene expression regulation, $\mathrm{R}$ supplementation did not modify FOXO1 mRNA expression, indicating that the important role of FOXO1 as a key transcription factor during decidualization was probably not affected (Kajihara et al. 2013). On the other hand, R supplementation reinforced the decreased expression of NOTCH1 and HOXA1O achieved by the DC in HESC; however, in t-HESC, R supplementation did not modify NOTCH1 and HOXA10 mRNA expression. The fact that the DC led to higher fold changes of HOXA10 and NOTCH1 in t-HESC than in HESC could explain why $\mathrm{R}$ supplementation did not further modify the expression of these genes in t-HESC (D vs ND for each cell type, Supplementary Fig. 2).

In this work, the increased expression of $P R L$ and IGFBP1 clearly indicates an increased level of differentiation of ESC and, furthermore, R supplementation seems to enhance the action of the DC during decidualization. Nevertheless, further experiments are needed to confirm in which degree the signaling pathways of the DC and R overlap and to

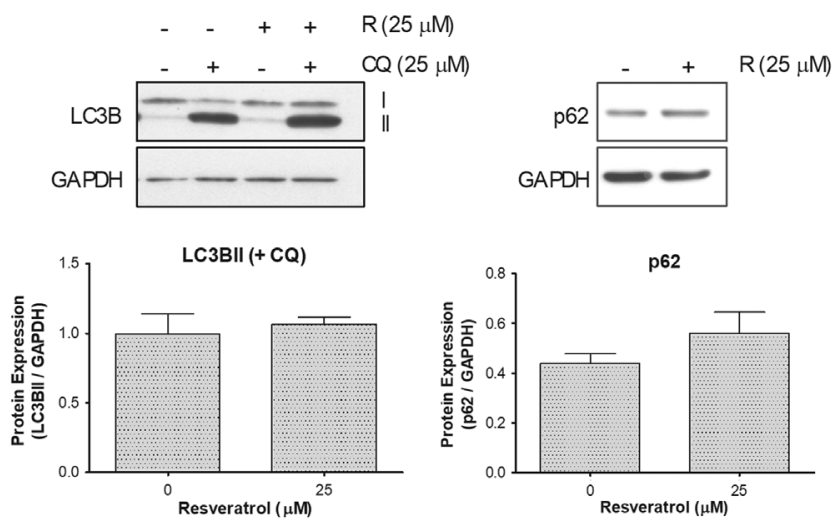

Figure 7 Effect of R supplementation over autophagic flux during t-HESC in vitro decidualization. t-HESC were treated with DC for 3 days and then treated with DC plus $25 \mu \mathrm{M} \mathrm{R}$ or vehicle for 2 more days (day 5$)$. CQ $(25 \mu \mathrm{M})$ was added $8 \mathrm{~h}$ before harvesting the cells. Figure shows representative immunoblots for protein levels of LC3B, p62 and GAPDH (internal control) $(n=4)$. Data represent mean relative protein expression to $\mathrm{GAPDH} \pm$ S.E.M. DC: decidualization cocktail; R: resveratrol; CQ: chloroquine.

define the DC-independent signaling pathways that are activated by $\mathrm{R}$ during decidualization.

\section{Resveratrol and cell cycle regulation}

Another important finding of our study is the fact that proliferation was slightly diminished by $R$ supplementation, reinforcing the reduction in cell proliferation stimulated by the DC. This effect of $\mathrm{R}$ on cell proliferation was more prominent in t-HESC than in HESC. It is expected that primary cells like HESC, with a limited lifespan, will be more sensitive to drugs affecting cell proliferation than immortalized cells like t-HESC. Particularly, in this work we saw that the fold change of KI67 in D vs ND cells was much lower in HESC than in $\mathrm{t}$-HESC. Therefore, it is not surprising that the effect of $\mathrm{R}$ over cell proliferation was more obvious in t-HESC, that maintain their proliferative capacity after the addition of the DC, than in HESC, that nearly completely lost KI67 expression during decidualization.

Going into detail of $\mathrm{R}$ regulation of cell proliferation and cell cycle progression, we found that the expression of positive cell cycle regulators, like CCNB1, CCND1 and different CDKs, was decreased during decidualization and further decreased in response to $\mathrm{R}$ supplementation. First, these results agree with previous studies describing a decreased expression of CCND1, CDK2 and CDC2 during ESC differentiation (Wang et al. 2018). And secondly, the fact that $R$ was able to decrease even further the expression of these genes suggests a clear reinforcement by $\mathrm{R}$ of the $\mathrm{DC}$ regulation of cell cycle progression. Finally, we observed an increased expression of CCNA1 in t-HESC and a decreased expression in HESC. CCNA1 has not been 
previously described in relation to decidualization, and its involvement in decidualization is still unclear after this study. Further experiments are needed to define if CCNA1 has a role during decidualization.

Regarding the expression of negative regulators of cell cycle progression, the expression of p21 was slightly decreased by the DC in t-HESC and was highly increased in HESC. This difference could also be explained by the different proliferative capacity of both cell types, suggesting again that HESC are more sensitive to the anti-proliferative effect of the DC than t-HESC.

Overall, we suggest that the resulted balance of positive and negative cell cycle regulators that was turned in favor of the inhibition of cell cycle progression by the DC was further reinforced by $\mathrm{R}$ supplementation (Supplementary Fig. 4) (Logan et al. 2012, Casimiro et al. 2014, Wang et al. 2018). We would like to highlight the fact that $\mathrm{R}$ affects ESC proliferation is an important effect to consider, since a defect in cell proliferation could affect decidua extension. However, as this effect was less prominent in HESC than in t-HESC, we would expect that low doses of $\mathrm{R}$ supplemented in vivo will drive the changes in cell cycle regulators needed for an improved decidualization without affecting decidua extension. Nevertheless, an optimal time and dose for $\mathrm{R}$ in vivo supplementation is still to be defined to guaranty its safe use in the preparation of the uterus for a successful pregnancy, contributing to a proper decidualization without affecting the pre-decidual proliferation.

\section{Resveratrol and endometrial health}

It has recently been published that $100 \mu \mathrm{M}$ of $\mathrm{R}$ inhibits in vitro decidualization (Ochiai et al. 2019). There are several differences between our experimental settings and the one described by Ochiai and colleagues. First, we used a lower range of $\mathrm{R}$ concentrations. Our highest concentration $(50 \mu \mathrm{M})$ is half the concentration with which they found a significant reduction in the expression of decidualization markers. Furthermore, if we pay attention to the $50 \mu \mathrm{M}$ dose (Figs 1 and $3 \mathrm{~A}$, B), we see that the expression of PRL and IFGBP1 starts to decrease compared to lower doses. Therefore, it is possible that much higher $\mathrm{R}$ concentrations would lead to a deleterious effect on decidualization. Second, we used a lower concentration of cAMP. cAMP helps to catalyze faster the decidualization reaction (Brar et al. 1997) and we believed, and confirmed with this work, that lower doses of cAMP are more effective to study the action of combined drugs as, in this study, $\mathrm{R}$ supplementation during decidualization. Finally, we administered $\mathrm{R}$ to pre-decidualized cells (day 3 of DC treatment) instead to ND cells.

Taken together the results presented by Ochiai and colleagues and our present findings, $\mathrm{R}$ supplementation seems to have a biphasic effect on decidualization, a positive outcome for cell differentiation at lower doses and a deleterious effect at higher doses. This biphasic effect of $\mathrm{R}$ has been previously described in adipocytes. In these cells, high doses of $\mathrm{R}(>20 \mu \mathrm{M})$ stimulate apoptosis and inhibit differentiation, while lower doses of $\mathrm{R}(1-10 \mu \mathrm{M})$ enhance in vitro adipocyte differentiation (Hu et al. 2015). It is worth to notice that for $\mathrm{R}$ action during adipocyte differentiation, the authors also utilized a weaker differentiation cocktail, as we described here for $\mathrm{R}$ supplementation during decidualization.

Interestingly, it has been also described that high doses of $\mathrm{R}$ are difficult to achieve physiologically (Boocock et al. 2007, Brown et al. 2010). Therefore, for the bench to bedside transition of $\mathrm{R}$ supplementation, it is important to define a physiologically achievable dose with a desirable effect for ESC function. In this same line of evidence are our present findings, as we were able to show that even the lower dose of R used $(6.25 \mu \mathrm{M})$ was effective to modified gene expression in ESC during decidualization.

Regarding the signaling pathways described by Ochiai and colleagues, they found that mRNA expression of $B T C 2$, an anti-proliferative gene, was increased during HESC decidualization and this increase was abrogated by $100 \mu \mathrm{M}$ of $\mathrm{R}$, indicating a positive regulation of cell proliferation upon $\mathrm{R}$ addition (Ochiai et al. 2019). This discrepancy with our results is expected as we are showing an opposite effect of $\mathrm{R}$ supplementation during decidualization. Furthermore, this result also supports the importance of focusing on proliferation and on the expression of cell cycle regulators while studying the effect of different drugs during decidualization. Besides, Ochiai and colleagues described that R-inhibitory action is mediated by SIRT1-dependent and SIRT1independet mechanisms (Ochiai et al. 2019). Finally, they suggest that the accelerated down-regulation of the CRABP2-RAR pathway by $\mathrm{R}$ additionally mediates R-inhibitory action during decidualization. However, further experiments are still needed to define the precise signaling pathways activated by $\mathrm{R}$ in its biphasic effect during ESC decidualization.

In conclusion, our present work suggests that $R$ supplementation could be beneficial for the correct decidualization of ESC. Further experiments are needed to define the signaling pathways activated by $R$ and, in particular, to define the optimal dose, time of administration and length of the treatment for achieving a beneficial effect during in vivo decidualization. We are positive that the findings of this work will lead to a better understanding of endometrial function and will facilitate the identification and selection of new therapeutic options for fertility-related endometrial pathologies.

\section{Supplementary materials}

This is linked to the online version of the paper at https://doi. org/10.1530/REP-19-0425. 


\section{Declaration of interest}

The authors declare that there is no conflict of interest that could be perceived as prejudicing the impartiality of the research reported.

\section{Funding}

A C M C postdoctoral position was fully supported by the Georg Forster Fellowship Program for Postdoctoral Researchers from the Alexander von Humboldt Foundation and by the Postdoctoral Fellowship from the IBSA Foundation for Scientific Research.

\section{Author contribution statement}

A C M C conceived the idea, designed and performed the experiments, analyzed the data and wrote the manuscript. A C M C, T S and A G provided funding. L L consented the patients. $\mathrm{T} \mathrm{S}$ and $\mathrm{A} G$ contributed to the final version of the manuscript. A G supervised work.

\section{Acknowledgements}

We thank the central laboratory of the University Hospital of Heidelberg.

\section{References}

Afshar Y, Miele L \& Fazleabas AT 2012 Notch1 is regulated by chorionic gonadotropin and progesterone in endometrial stromal cells and modulates decidualization in primates. Endocrinology 153 2884-2896. (https://doi.org/10.1210/en.2011-2122)

Aghajanova L, Hamilton A, Kwintkiewicz J, Vo KC \& Giudice LC 2009 Steroidogenic enzyme and key decidualization marker dysregulation in endometrial stromal cells from women with versus without endometriosis. Biology of Reproduction 80 105-114. (https://doi. org/10.1095/biolreprod.108.070300)

Boocock DJ, Faust GES, Patel KR, Schinas AM, Brown VA, Ducharme MP, Booth TD, Crowell JA, Perloff M, Gescher AJ et al. 2007 Phase I dose escalation pharmacokinetic study in healthy volunteers of resveratrol, a potential cancer chemopreventive agent. Cancer Epidemiology, Biomarkers and Prevention 16 1246-1252. (https://doi. org/10.1158/1055-9965.EPI-07-0022)

Brar AK, Frank GR, Kessler CA, Cedars MI \& Handwerger S 1997 Progesterone-dependent decidualization of the human endometrium is mediated by cAMP. Endocrine 6 301-307. (https://doi.org/10.1007/ BF02820507)

Brighton PJ, Maruyama Y, Fishwick K, Vrljicak P, Tewary S, Fujihara R, Muter J, Lucas ES, Yamada T, Woods L et al. 2017 Clearance of senescent decidual cells by uterine natural killer cells in cycling human endometrium. eLife 6 1-23. (https://doi.org/10.7554/eLife.31274)

Brosens IJ, Takeda S, Acevedo CH, Lewis MP, Kirby PL, Symes EK, Krausz T, Purohit A, Gellersen B \& White JO 1996 Human endometrial fibroblasts immortalized by simian virus 40 large $\mathrm{T}$ antigen differentiate in response to a decidualization stimulus. Endocrinology 137 2225-2231. (https:// doi.org/10.1210/endo.137.6.8641169)

Brown VA, Patel KR, Viskaduraki M, Crowell JA, Perloff M, Booth TD, Vasilinin G, Sen A, Schinas AM, Piccirilli G et al. 2010 Repeat dose study of the cancer chemopreventive agent resveratrol in healthy volunteers: safety, pharmacokinetics, and effect on the insulin-like growth factor axis. Cancer Research 70 9003-9011. (https://doi.org/10.1158/00085472.CAN-10-2364)

Casimiro MC, Velasco-Velázquez M, Aguirre-Alvarado C \& Pestell RG 2014 Overview of cyclins D1 function in cancer and the CDK inhibitor landscape: past and present. Expert Opinion on Investigational Drugs 23 295-304. (https://doi.org/10.1517/13543784.2014.867017)

Cucciolla V, Borriello A, Oliva A, Galletti P, Zappia V \& Della Ragione F 2007 Resveratrol: from basic science to the clinic. Cell Cycle 6 2495-2510. (https://doi.org/10.4161/cc.6.20.4815)

De Oliveira LG, Lash GE, Murray-Dunning C, Bulmer JN, Innes BA, Searle RF, Sass N \& Robson SC 2010 Role of interleukin 8 in uterine natural killer cell regulation of extravillous trophoblast cell invasion. Placenta 31 595-601. (https://doi.org/10.1016/j.placenta.2010.04.012)

Delmas D, Solary E \& Latruffe N 2011 Resveratrol, a phytochemical inducer of multiple cell death pathways: apoptosis, autophagy and mitotic catastrophe. Current Medicinal Chemistry 18 1100-1121. (https://doi.org/10.2174/092986711795029708)

Feoktistova M, Geserick P \& Leverkus M 2016 Crystal violet assay for determining viability of cultured cells. Cold Spring Harbor Protocols 2016 pdb.prot087379. (https://doi.org/10.1101/pdb.prot087379)

Garrido-Gomez T, Dominguez F, Quiñonero A, Diaz-Gimeno P, Kapidzic M, Gormley M, Ona K, Padilla-Iserte P, McMaster M, Genbacev O et al. 2017 Defective decidualization during and after severe preeclampsia reveals a possible maternal contribution to the etiology. PNAS 114 E8468-E8477. (https://doi.org/10.1073/pnas.1706546114)

Gellersen B \& Brosens J 2003 Cyclic AMP and progesterone receptor cross-talk in human endometrium: a decidualizing affair. Journal of Endocrinology 178 357-372. (https://doi.org/10.1677/joe.0.1780357)

Gellersen B \& Brosens JJ 2014 Cyclic decidualization of the human endometrium in reproductive health and failure. Endocrine Reviews 35 851-905. (https://doi.org/10.1210/er.2014-1045)

Godbole G, Suman P, Malik A, Galvankar M, Joshi N, Fazleabas A, Gupta SK \& Modi D 2017 Decrease in expression of HOXA10 in the decidua after embryo implantation promotes trophoblast invasion. Endocrinology 158 2618-2633. (https://doi.org/10.1210/en.2017-00032)

Hannan NJ, Brownfoot FC, Cannon P, Deo M, Beard S, Nguyen TV, Palmer KR, Tong S \& Kaitu'U-Lino TJ 2017 Resveratrol inhibits release of soluble fms-like tyrosine kinase (sFlt-1) and soluble endoglin and improves vascular dysfunction - implications as a preeclampsia treatment. Scientific Reports 7 1819. (https://doi.org/10.1038/s41598017-01993-w)

Hasima N \& Ozpolat B 2014 Regulation of autophagy by polyphenolic compounds as a potential therapeutic strategy for cancer. Cell Death and Disease 5 e1509. (https://doi.org/10.1038/cddis.2014.467)

Hu P, Zhao L \& Chen J 2015 Physiologically achievable doses of resveratrol enhance 3T3-L1 adipocyte differentiation. European Journal of Nutrition 54 569-579. (https://doi.org/10.1007/s00394-014-0738-4)

Kajihara T, Brosens JJ \& Ishihara O 2013 The role of FOXO1 in the decidual transformation of the endometrium and early pregnancy. Medical Molecular Morphology 46 61-68. (https://doi.org/10.1007/s00795-0130018-z)

Klemmt PAB, Carver JG, Kennedy SH, Koninckx PR \& Mardon HJ 2006 Stromal cells from endometriotic lesions and endometrium from women with endometriosis have reduced decidualization capacity. Fertility and Sterility 85 564-572. (https://doi.org/10.1016/j.fertnstert.2005.08.046)

Klionsky DJ, Abdelmohsen K, Abe A, Abedin MJ, Abeliovich H, Arozena AA, Adachi H, Adams CM, Adams PD, Adeli K et al. 2016 Guidelines for the use and interpretation of assays for monitoring autophagy (3rd editio). Autophagy 12 1-222. (https://doi.org/10.1080/15548627.2015.1100356)

Ko JH, Sethi G, Um JY, Shanmugam MK, Arfuso F, Kumar AP, Bishayee A \& Ahn KS 2017 The role of resveratrol in cancer therapy. International Journal of Molecular Sciences 18 1-36. (https://doi.org/10.3390/ijms18122589)

Kolahdouz Mohammadi R \& Arablou T 2017 Resveratrol and endometriosis: in vitro and animal studies and underlying mechanisms (Review). Biomedicine and Pharmacotherapy 91 220-228. (https://doi. org/10.1016/j.biopha.2017.04.078)

Krikun G, Mor G, Alvero A, Guller S, Schatz F, Sapi E, Rahman M, Caze R, Qumsiyeh M \& Lockwood CJ 2004 A novel immortalized human endometrial stromal cell line with normal progestational response. Endocrinology 145 2291-2296. (https://doi.org/10.1210/en.2003-1606)

Kwon M, Kim JM, Lee K, Park SY, Lim HS, Kim T \& Jeong D 2016 Synchronized cell cycle arrest promotes osteoclast differentiation. International Journal of Molecular Sciences 17 1-8. (https://doi.org/10.3390/ijms17081292)

Liu M, Yin Y, Ye X, Zeng M, Zhao Q, Keefe DL \& Liu L 2013 Resveratrol protects against age-associated infertility in mice. Human Reproduction 28 707-717. (https://doi.org/10.1093/humrep/des437) 
Livak KJ \& Schmittgen TD 2001 Analysis of relative gene expression data using real-time quantitative PCR and the 2- $\triangle \triangle C T$ method. Methods 25 402-408. (https://doi.org/10.1006/meth.2001.1262)

Logan PC, Steiner M, Ponnampalam AP \& Mitchell MD 2012 Cell cycle regulation of human endometrial stromal cells during decidualization. Reproductive Sciences 19 883-894. (https://doi. org/10.1177/1933719112438447)

Mestre Citrinovitz AC, Strowitzki T \& Germeyer A 2019 Decreased autophagy impairs decidualization of human endometrial stromal cells: a role for ATG proteins in endometrial physiology. International Journal of Molecular Sciences 20 3066. (https://doi.org/10.3390/ijms20123066)

Myster DL \& Duronio RJ 2000 Cell cycle: to differentiate or not to differentiate? Current Biology 10 R302-R304. (https://doi.org/10.1016/ S0960-9822(00)00435-8)

Ochiai A, Kuroda K, Ozaki R, Ikemoto Y, Murakami K, Muter J, Matsumoto A, Itakura A, Brosens JJ \& Takeda S 2019 Resveratrol inhibits decidualization by accelerating downregulation of the CRABP2-RAR pathway in differentiating human endometrial stromal cells. Cell Death and Disease 10 276. (https://doi.org/10.1038/s41419-019-1511-7)

Ortega I \& Duleba AJ 2015 Ovarian actions of resveratrol. Annals of the New York Academy of Sciences 1348 86-96. (https://doi.org/10.1111/ nyas.12875)

Pitman H, Innes BA, Robson SC, Bulmer JN \& Lash GE 2013 Altered expression of interleukin-6, interleukin-8 and their receptors in decidua of women with sporadic miscarriage. Human Reproduction $\mathbf{2 8}$ 2075-2086. (https://doi.org/10.1093/humrep/det233)

Qian K, Chen H, Wei Y, Hu J \& Zhu G 2005 Differentiation of endometrial stromal cells in vitro: down-regulation of suppression of the cell cycle inhibitor p57 by HOXA10? Molecular Human Reproduction 11 245-251. (https://doi.org/10.1093/molehr/gah147)

Ramathal CY, Bagchi IC, Taylor RN \& Bagchi MK 2010 Endometrial decidualization: of mice and men. Seminars in Reproductive Medicine 28 17-26. (https://doi.org/10.1055/s-0029-1242989)

Reichert M \& Eick D 1999 Analysis of cell cycle arrest in adipocyte differentiation. Oncogene 18 459-466. (https://doi.org/10.1038/ sj.onc.1202308)

Ricci AG, Olivares CN, Bilotas MA, Bastón JI, Singla JJ, Meresman GF \& Barañao RI 2013 Natural therapies assessment for the treatment of endometriosis. Human Reproduction 28 178-188. (https://doi. org/10.1093/humrep/des369)

Ruijtenberg S \& van den Heuvel S 2016 Coordinating cell proliferation and differentiation: antagonism between cell cycle regulators and cell typespecific gene expression. Cell Cycle 15 196-212. (https://doi.org/10.108 0/15384101.2015.1120925)

Soufi A \& Dalton S 2016 Cycling through developmental decisions: how cell cycle dynamics control pluripotency, differentiation and reprogramming. Development 143 4301-4311. (https://doi.org/10.1242/ dev.142075)

Strowitzki T, Germeyer A, Popovici R \& von Wolff M 2006 The human endometrium as a fertility-determining factor. Human Reproduction Update 12 617-630. (https://doi.org/10.1093/humupd/dml033)

Takano M, Lu Z, Goto T, Fusi L, Higham J, Francis J, Withey A, Hardt J, Cloke B, Stavropoulou AV et al. 2007 Transcriptional cross talk between the forkhead transcription factor forkhead box O1A and the progesterone receptor coordinates cell cycle regulation and differentiation in human endometrial stromal cells. Molecular Endocrinology 21 2334-2349. (https://doi.org/10.1210/me.2007-0058)

Wang L, Yang H, Hu L, Hu D, Ma S, Sun X, Jiang L, Song J, Ji L, Masau JF et al. 2018 CDKN1C (P57): one of the determinants of human endometrial stromal cell decidualization. Biology of Reproduction 98 277-285. (https://doi.org/10.1093/biolre/iox187)

Zou Y, Zuo Q, Huang S, Yu X, Jiang Z, Zou S, Fan M \& Sun L 2014 Resveratrol inhibits trophoblast apoptosis through oxidative stress in preeclampsia-model rats. Molecules 19 20570-20579. (https://doi. org/10.3390/molecules191220570)

Received 5 September 2019

First decision 30 September 2019

Revised manuscript received 14 January 2020

Accepted 28 January 2020 